\title{
Thermonuclear Excitation of $R$-modes in Neutron Stars
}

\author{
Umin Lee \\ Laboratoire de Physique, Ecole Normale Superieure de Lyon, 69364 \\ Lyon Cedex or
}

Tod E. Strohmayer

Code 666, NASA/Goddard Space Flight Center, Greenbelt, MD 20771

\begin{abstract}
Neutron stars display phenomena which result from the burning of accreted nuclear fuel in their surface layers. In addition to producing X-ray bursts and other temporal phenomema, such burning can excite oscillation modes of the neutron star. Here we investigate the stability of $R$-modes in uniformly rotating neutron stars using nonadiabatic pulsation calculations of steady burning envelopes on accreting neutron stars. We find that the fundamental $R$-modes are unstable for a large range of accretion rates, and thus might manifest themselves observationally in the power spectra of accreting sources above about $1 \mathrm{kHz}$.
\end{abstract}

\section{1. $R$-modes oscillations in rotating neutron stars}

Sensitive searches in LMXB have yet to reveal the pulsation signal of the rapidly rotating neutron star commonly thought to reside in these systems (Wood et al. 1991). Detection of such pulsations would firmly establish the hypothesized evolutionary link between these systems and the millisecond pulsars. The ability to observe rotationally induced pulsations depends on the degree to which these systems can produce intensity variations on the neutron star, for example, an accretion hotspot, or flux asymmetry caused by the magnetic field. If the magnetic fields in these systems are incapable of producing an observable rotational modulation, then what other physical processes can produce asymmetries, or patterns, which might be modulated at small, but detectable levels by stellar rotation? One possibility is that surface waves or oscillations can be excited to sufficient amplitude to produce observable modulations. Indeed, there are several reports in the literature of periodic intensity variations which occurred either during or in the vicinity of an X-ray burst (Livio \& Bath 1982). At present there is no widely accepted scenario which explains all of the reported periodicities, but one possibility is that some of these periodic variations are due to surface oscillations. A potentially interesting class of such oscillations are the $R$-modes (also called planetary waves or Rossby waves), whose frequencies are closely related to the spin frequency of the star (Saio 1982). These modes reside largely in the surface layers of a neutron star, and involve motions of a small fraction of the total stellar mass. They therefore require less energy to excite to potentially observable amplitudes than more global modes. Our nonadiabatic 
calculations are performed using surface envelopes which have been constructed using the thin shell formalism described by Paczynski (1970). Hydrogen burning in accreting systems occurs in the steady-state for a variety of accretion rates, so for these calculations we assume that the shell steadily burns fuel at a rate equal to the accretion rate. We have investigated a range of accretion rates, however, here we report only results for a single illustrative case with $\dot{M}=0.8 \dot{M}_{\text {edd }}$.

In the lowest order approximation, $R$-modes are toroidal modes with oscillation frequency $\sigma_{0}=2 m \Omega / l(l+1)$, where $\Omega$ is the angular rotation frequency of the star and $l$ is the spherical harmonic index. We investigate the pulsational stability of $R$-modes using the nonadiabatic formulation for uniformly rotating stars described by Lee \& Saio (1987). In this treatment the displacement vector of an oscillation mode is expanded as an infinite series of spherical harmonics with constant azimuthal index $m$. The results of our four term, nonadiabatic calculations are a series of complex eigenfrequencies which describe as a function of rotation period the oscillation period in the rotating reference frame and the excitation or decay timescale. In Table 1 we have compiled the results for the $m=-1$, fundamental $R$-mode (denoted $r_{0}$ ) for three values of the rotation period of the neutron star. Shown are the rotation period, the $R$-mode periods in the rotating and inertial frames (in msec), and the growth rate $\mu$ for each mode. The large, positive growth rates indicate that these modes are strongly unstable to hydrogen burning.

Table 1. Periods and Growth Rates for $R$-modes $(\dot{M}=0.8)$

\begin{tabular}{ccccc} 
Modes & $\boldsymbol{P}_{\text {spin }}$ & $\Pi_{\text {rot }}$ & $\Pi_{\text {inert }}$ & $\mu$ \\
\hline$r_{0}$ & 46.09 & 175.9 & 62.46 & 0.042 \\
$r_{0}$ & $\mathbf{9 . 2 1 9}$ & 125.6 & 9.949 & 0.070 \\
$r_{0}$ & $\mathbf{4 . 6 0 9}$ & 120.5 & $\mathbf{4 . 7 9 3}$ & 0.061
\end{tabular}

\section{Discussion}

We find that the fundamental $R$-modes are unstable to the nuclear burning which occurs in the accreted surface layers of neutron stars. The large growth rates we find suggest that these modes may be driven to sufficient amplitudes to become observationally significant. The expected mode periods indicate that searches in the X-ray by future missions such as XTE be optimized in the $\mathrm{kHz}$ region of the power spectrum.

\section{References}

Lee, U., \& Saio, H. 1987, MNRAS, 225, 643

Livio, M., \& Bath, G. T. 1982, A\&A, 116, 286

Paczynski, B. 1970, Acta Astro., 20, 287

Saio, H. 1982, ApJ, 256, 717

Wood, K. S., et al. 1991, ApJ, 379, 295 